\title{
AVALIAÇÃO DAS QUALIDADES HIGIÊNICOSSANITÁRIAS DAS UNIDADES DE ALIMENTAÇÃO DAS ESCOLAS MUNICIPAIS E CEMEI DO CARMO DO RIO VERDE-GO
}

\author{
EVALUATION OF THE HYGIENIC SANITARY QUALITIES OF THE \\ FOOD UNITS OF THE MUNICIPAIS E SCHOOLS CEMEI OF CARMO DO \\ RIO VERDE-GO
}

\author{
Me. Gilmar Aires da Silva \\ Professor Mestre do curso de Farmácia - Faculdade Evangélica de Ceres, Ceres-GO, Brasil \\ E-mail: gilmaraires@gmail.com
}

\section{Carla Cristina Gomes}

Acadêmica do curso de Farmácia - Faculdade Evangélica de Ceres, Ceres-GO, Brasil

E-mail: carla-cristyna@ hotmail.com

\section{Millena da Silva Coelho}

Acadêmica do curso de Farmácia - Faculdade Evangélica de Ceres, Ceres-GO, Brasil

E-mail: millena.coelho@gmail.com

\section{Endereço para correspondência:}

Av. Brasil, S/N, Qd. 13, Setor Morada Verde Ceres - GO. CEP: 763000-000

Fone: (62) 3323-1040;

E-mail: gilmaraires@gmail.com

RESUMO: Introdução: A alimentação de qualidade é muito importante na vida do ser humano especialmente por ajudar a manter a saúde do indivíduo, portanto a alimentação escolar tendo como base o PNAE contribui significativamente nas condições nutricionais de crianças e jovens, mas para isso é preciso que os manipuladores dos alimentos e o ambiente estejam capacitados a realizar manipulação dos alimentos segundo as RDC/ANVISA $n^{\circ}$ 216/2004 e n²75/2002 que tratam das condições higiênicossanitárias e Boas Práticas de fabricação dos alimentos. Objetivos: avaliar a qualidade higiênicossanitária dos manipuladores, utensílios, ambiente e alimentos que são servidos nas escolas e CEMEI da rede municipal de ensino da cidade de Carmo do Rio Verde-GO e confrontar os dados coletados, com os dados propostos nas normas de vigilância sanitária. Metodologia: estudo de campo de caráter qualitativo e quantitativo que foi realizado em quatro escolas na área urbana de Carmo do Rio Verde-GO, com aplicação de check-list. Resultados e discussão: Os resultados indicam mais de $60 \%$ de conformidades nas unidades avaliadas. As instituições se enquadram no grupo 2 conforme padrão estabelecido pela RDC n 275 de 21 de outubro de 2002 da ANVISA/MS na qual se tem de 50 a $75 \%$ de atendimento aos itens. Conclusão: De acordo com os resultados obtidos nesse estudo, fica claro que existem falhas na qualidade higiênicossanitária da merenda escolar das instituições pesquisadas, são necessários medidas corretivas em relação às instalações, manipuladores, equipamentos e utensílios para que se atenda as recomendações das RDC 275 E 216.

Palavras-Chave:; Alimentação; alimentação de qualidade, manipulador. 
ABSTRACT: Introduction: Quality food is very important in the life of the human being especially for helping to maintain the health of the individual, so school feeding based on the PNAE contributes significantly to the nutritional conditions of children and young people, but for this it is necessary that the manipulators of the food and the environment are able to handle food according to DRC / ANVISA n ${ }^{\circ}$ 216/2004 and n ${ }^{\circ}$ 275/2002 dealing with sanitary and sanitary conditions and Good Manufacturing Practices. Objectives: To evaluate the hygienic and sanitary quality of the handlers, utensils, environment and food that are served in the schools and CEMEI located in the urban area of the municipal education network of the city of Carmo do Rio Verde-GO and to compare the data collected, with the data proposed in the standards of health surveillance. Methodology: a qualitative and quantitative field study that was carried out in four schools in the urban area of Carmo do Rio Verde-GO, with check-list application. Results and discussion: The results indicate more than $60 \%$ of conformities in the evaluated units. The institutions fall into group 2 according to the standard established by RDC $n^{\circ} 275$ of October 21, 2002 of ANVISA / MS in which there is 50 to $75 \%$ of attendance to items. Conclusion: According to the results obtained in this study, it is clear that there are deficiencies in the sanitary and sanitary quality of the school meals of the researched institutions, corrective measures are necessary in relation to the facilities, manipulators, equipment and utensils to meet the recommendations of the RDC 275 E 216.

Keyword: Feeding; quality feed, manipulator.

\section{INTRODUÇÃO}

A primeira infância, é a fase em que a criança apresenta elevadas necessidades de energia e nutrientes devido ao rápido desenvolvimento motor e psíquico. Dessa forma, é necessário fornecer uma alimentação adequada para assegurar o crescimento, desenvolvimento e que promova o seu bem estar. A alimentação de qualidade exerce um papel muito importante na vida do ser humano, especialmente ajudando a manter a saúde no indivíduo. Para que a alimentação cumpra seu papel na promoção da saúde é necessário que seja feita uma dieta equilibrada, adequada nutricionalmente e que forneça todos os nutrientes essenciais ao corpo possibilitando o bom funcionamento do organismo (RIEKES, 2004; FERREIRA, SANTOS e RAVAZZANI, 2017).

A alimentação escolar pode contribuir significativamente para a melhoria das condições nutricionais de crianças e jovens, diminuindo deficiências nutricionais e outros problemas relacionados à alimentação inadequada, melhorando consideravelmente o desempenho escolar, favorecendo o crescimento e o desenvolvimento, visto que para muitos desses jovens e crianças a alimentação escolar é a principal refeição durante o dia. Representa também um importante fator de desenvolvimento econômico local (ACCIOLY, 2009). 
A desnutrição é um problema de saúde pública que causa grandes repercussões, principalmente em crianças, pois afeta todos os sistemas diminuindo a qualidade de vida, impedindo o crescimento e desenvolvimento adequados, o que faz com as chances dessas crianças de se tornarem adultos saudáveis sejam restringidas consideravelmente. Umas das respostas iniciais a carência nutricional é a perda de peso, seguida da desaceleração do crescimento, alterações hormonais, enzimáticas e da resposta imunológica. Devido a esses fatores, as crianças desnutridas são mais susceptíveis a infecções, diarréias, distúrbios eletrolíticos, hipoglicemia e hipotermia. A anemia é um agravante da desnutrição e pode aparecer em consequência a carências específicas como a de ferro como também aparece em resposta à baixa demanda de oxigênio pelos tecidos (GOULART; VIANA, 2008).

Gerenciado pelo Fundo Nacional de Desenvolvimento da Educação do Ministério da Educação (FNDE/MEC), o Programa Nacional de Alimentação Escolar (PNAE) tendo por base a perspectiva do direito humano à alimentação, é referência mundial na área de alimentação escolar por atender a alunos matriculados na educação infantil e ensino fundamental das escolas públicas e escolas filantrópicas de todo país. Uma das responsabilidades primordiais do centro municipal de educação infantil (CMEI) é a alimentação que permite a criança um bom desenvolvimento físico e intelectual, evitando possíveis deficiências nutricionais (VOOS, 2009: SILVA; OLIVEIRA e SANTOS, 2012 ).

O PNAE atende anualmente a milhares de alunos que frequentam creches, préescolas e escolas do ensino fundamental das redes federal, estadual e municipal. Devido a essa ampla abrangência, o controle higiênicossanitário no ambiente escolar é um aspecto que deve ser observado e discutido afim de que os padrões de qualidade sejam alcançados garantindo alimentos seguros e evitando às doenças transmitidas por alimentos (DTA) que são um grande risco para as crianças devido ao fato de serem mais susceptíveis por não possuírem o sistema imunológico totalmente desenvolvido (DA SILVA, 2010).

Os programas de alimentação escolar ofertam um grande número de refeições e por isso oferecem grandes riscos de contaminação pelo desenvolvimento bacteriano em alimentos. Conhecer as condições higiênicossanitárias na produção de alimentos é indispensável, pois isso interfere diretamente na qualidade do alimento que está sendo preparado. O manipulador de alimentos pode, sem dúvida, interferir na qualidade do alimento e por isso é importante que ele faça treinamento na prevenção de enfermidades transmitidas por alimentos (OLIVEIRA et. al. 2008). 
Segundo as RDC/ANVISA $n^{\circ} 216 / 2004$ e $n^{\circ} 275 / 2002$, que tratam das condições higiênicossanitárias e boas práticas de manipulação e fabricação, os manipuladores de alimento devem realizar cursos de capacitação em que sejam abordados temas como: doenças transmitidas por alimentos, manipulação higiênica dos alimentos, contaminantes alimentares e boas práticas. A presença de um profissional nutricionista no local de manipulação está intrinsecamente ligada na alta adequação nos locais de manipulação de alimentos.

Os equipamentos e utensílios são as principais ferramentas de trabalho em um serviço de alimentação, faz-se necessário uma correta higienização e manutenção periódica. A higienização deve fazer parte do esquema de segurança sanitária da unidade nutricional. A higienização dos utensílios, equipamentos, estabelecimento e também dos manipuladores é de fundamental importância na garantia da segurança dos alimentos (HENRIQUES, 2014).

O alimento que apresenta uma característica alterada caracteriza-se como alimento contaminado, pois, os alimentos são excelentes substratos onde se desenvolvem numerosas espécies e variedades de microrganismos, por vários fatores ambientais. As bactérias são os microrganismos de maior participação nos processos de contaminação de alimentos, pois atuam em diferentes ambientes. A contaminação do alimento pode ocorrer tanto pelo contato direto do manipulador no alimento ou por outros fatores com condições inadequadas para que isso ocorra (temperatura, instalações, utensílios e equipamentos mal cuidados) (MULLER, 2011).

Durante o processo de transmissão de microorganismos para os alimentos, o homem é um importante veículo, podendo ser o contaminante quando apresenta processos infecciosos, se encontra em período de convalescença ou é portador assintomático. Além de a contaminação ocorrer pelos processos de produção, ela ocorre também durante a distribuição e armazenamento do alimento por diversos fatores. A fim de evitar a veiculação de microorganismos patogênicos, deve-se controlar a contaminação, a multiplicação e a sobrevivência microbiana nos diversos ambientes, tais como móveis, utensílios, equipamentos e manipuladores (VILA, SILVEIRA, ALMEIDA, 2014).

Diante desse contexto, este estudo se propôs a analisar o ambiente de produção e distribuição do alimento em um CEMEI e três escolas municipais de Carmo do Rio VerdeGO, a fim de verificar seus aspectos higiênicossanitários e ambientais, observando a produção e o destino dado aos resíduos oriundos da preparação da merenda escolar. A importância do estudo se dá pela necessidade de conhecer as condições em que a alimentação escolar é 
produzida e disponibilizada aos estudantes, tendo em vista que, para garantir a segurança alimentar são considerados vários aspectos como instalações, utensílios, equipamentos, manipulador, e até mesmo a qualidade do alimento que chega para a produção.

\section{METODOLOGIA}

2

Trata-se de um estudo de campo de caráter qualitativo e quantitativo que foi realizado no período de agosto a setembro de 2017 em três escolas e um CMEI da Rede Municipal de Ensino do Carmo do Rio Verde-GO. Onde são servidas refeições à todas as crianças diariamente. Cada escola foi identificada, como A, B, C e D. As avaliações das condições higiênicossanitárias foram de caráter observacional através de um check-list elaborado segundo a RDC No275 de 2002.

Foram utilizados os critérios de classificação segundo a RDC 275 para avaliação dos dados obtidos nos check-list aplicado nos estabelecimentos, que são: no grupo 1 estarão aqueles que atenderem de 76 a 100\% dos itens, e serão considerados bom; no grupo 2 os que estiverem entre 51 a $75 \%$ de atendimento dos itens serão considerados regular e no grupo 3 os que estiverem de 0 a $50 \%$ de atendimentos dos itens serão considerados ruins.

A pesquisa foi aplicada as escolas mediante a assinatura do termo de consentimento livre e esclarecido pelo diretor de cada escola e também pela secretária de educação. Todas as escolas da área urbana da rede municipal de ensino do Carmo do Rio Verde participaram da pesquisa. Foram observadas também as condições higiênicossanitárias do ambiente e dos manipuladores.

\section{RESULTADO E DISCUSSÃO}

O gráfico 1 apresenta os dados referentes as conformidades e não conformidades de acordo com a RDC 275 em quatro escolas e um CEMEI municipais da cidade de Carmo do Rio Verde-GO. 


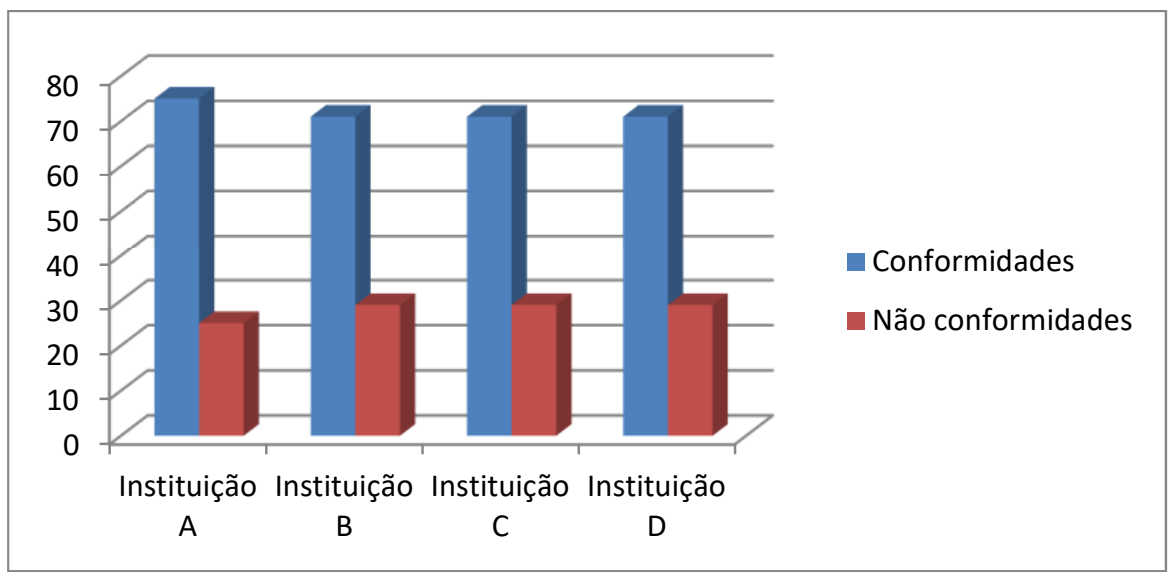

Gráfico 1: Conformidades e não conformidades de acordo com a RDC 275 das instituições de ensino municipais da cidade de Carmo do Rio Verde-GO.

Todas as instituições pesquisadas foram enquadradas no grupo dois, conforme padrão estabelecido pela RDC no 275 de 21 de outubro de 2002 da ANVISA/MS (AGÊNCIA NACIONAL DE VIGILÂNCIA SANITÁRIA, 2002), na qual se tem de 50 a $75 \%$ de atendimento aos itens (gráfico 1).

Nas Unidades de Alimentação e Nutrição (UAN) avaliadas as refeições são produzidas por dois manipuladores em cada instituição, estes possuem capacitação em boas práticas de manipulação de alimentos, mas sem atualizações períodicas. A implementação das Boas Práticas de Manipulação dos alimentos é uma das táticas utilizadas para se atingir um alto padrão de qualidade alimentar e tem como objetivo estabelecer procedimentos que sejam adequados e que contribuam com as condições higiênicossanitárias dos alimentos (SILVA, 2015).

Uma das inconformidades encontradas foi em relação ao uso dos EPIs, foi observado que o único EPI utilizados pelos manipuladores em todas as instituições avaliadas é a touca. A falta do uso ou o uso incorreto desses EPIs é considerado um meio de contaminação preocupante visto que os microrganismos podem ser transportados dos manipuladores para os alimentos. A contaminação alimentar está relacionada a ausência de conhecimento ou à negligência dos manipuladores e por isso o treinamento relacionado as Boas Práticas de Fabricação é um pré-requisito importante para que se possa alcançar a inocuidade dos alimentos (DEON, 2012). A contaminação do alimento pelo manipulador causa grande parte das toxi-infecções alimentares, portanto a educação e treinamento constante dos manipuladores de alimentos interferem positivamente na manutenção da qualidade higiênicossanitária dos produtos alimentícios (GOÉS et. al. 2001). 
Outra inconformidade observada foi às condições físicas de conservação do ambiente de preparo dos alimentos. As instituições B e D não possuem ralos sanfonados, nas quatro instituições pesquisadas as janelas e portas não possuem telas milimetradas, as lâmpadas não possuem proteção, as lixeiras não possuem pedais. As paredes da instituição C não possuem azulejos e encontram em mau estado de conservação, apresentando pontos com mofos e sujidades impregnadas. A fim de evitar a presença de insetos e roedores no local de armazenamento do alimento é preciso que portas e janelas tenham telas de proteção, porem a área deve ser bem ventilada para que assim a conservação dos produtos alimentares seja garantida e odores sejam evitados (STEFANELLO, LINN, MESQUITA, 2009).

Os utensílios que são utilizados em todas as instituições pesquisadas, são de plástico, alumínio e em aço inox, estando em boas condições de uso, nenhuma das UANs possui bancada apropriada para preparo de alimentos. Os mesmos são manipulados sobre bancadas de pias e tanques e mesas de madeira coberta por plástico, a madeira não é apropriada para o preparo de alimentos, pois é um meio que facilita o crescimento de microrganismos. Para atender a legislação em vigor (BRASIL, 2001) é preciso evitar a contaminação, multiplicação e a sobrevivência de microorganismos em diferentes locais, como: equipamentos, utensílios e manipuladores, contribuindo com a produção de alimentos de boa qualidade microbiológica além de não colocar a saúde dos usuários em risco com a veiculação de microrganismos patogênicos (BARROS, 2014).

Outra inconformidade encontrada em todas as instituições avaliadas foi as instalações sanitárias, nenhuma possui sabonete liquido inodoro, as toalhas para a secagem das mãos são de tecido. Resultados semelhantes também foram encontrados por Souza (2009).

Em relação ao controle das temperaturas, nenhuma das UANs realiza o controle das temperaturas nos freezers e geladeiras, a temperatura também não é aferida nos ambientes em que se manipulam os alimentos. A avaliação da temperatura é um dos parâmetros mais importantes na atividade bioquímica dos microrganismos, pois grande parte dos casos de DTAs está relacionada com a falta de controle na aferição da temperatura de conservação dos alimentos (ROCHA, 2010).

\section{CONCLUSÕES}

De acordo com os resultados obtidos nesse estudo, fica claro que existem falhas na qualidade higiênicossanitária no preparo da merenda escolar das instituições pesquisadas, REFACER v 7. , n. 1, 2018. ISSN - 2317-1367 
1 são necessários medidas corretivas em relação às instalações, manipuladores, equipamentos e

utensílios, para que atendam as recomendações das RDC 275 E 216 e possam assim ter uma garantia de boa qualidade higiênicossanitária da alimentação oferecida nas instituições. Tornase evidente que as cozinhas das instituições pesquisadas são cozinhas domésticas adaptadas, sendo assim nenhuma possui estrutura física adequada. Conclui-se que existem falhas em relação às documentações, nenhuma das instituições pesquisadas possui as mesmas expostas. É necessário que se tenha ajustes em relação à documentação e que seja elaborado e implantado em todas as instituições o manual de boas praticas de fabricação e manipulação dos alimentos e que os manipuladores sejam capacitados de acordo com as Boas Praticas para que se possam ter refeições produzidas com o Maximo de segurança alimentar e o mínimo possível de risco para os alunos.

\section{REFERÊNCIAS}

ACCIOLY, Elizabeth. A escola como promotora da alimentação saudável. Ciência em Tela, Vol. 2. 2009.

BARROS, Fabrício Maximiliano de Oliveira et al. Avaliação da presença de microorganismos patogênicos em alimentos servidos em restaurantes e escolas públicas do Município de Manaquiri, Amazonas. 2014.

DA SILVA, Cleliani de Cassia. Segurança Alimentar no Ambiente Escolar. Estratégias de Promoção da Saúde do Escolar: Atividade Física e Alimentação Saudável, p. 4. 2010

DE ANDRADE, NÉLIO JOSÉ; DA SILVA, ROSÁLIA MARIA MOREIRA; BRABES, Kelly Cristina Silva. Avaliação das condições microbiológicas em unidades de alimentação e nutrição. 2003.

\section{DEON, Barbara Cecconi. DIAGNÓSTICO DE BOAS PRÁTICAS DE ALIMENTAÇÃO EM} DOMICÍLIOS DA CIDADE DE SANTA MARIA-RS. Rio Grande do, 2012.

FERREIRA, Daiane Cristina; SANTOS, Michele Aparecida dos; RAVAZZANI, Edilcéia Domingues do Amaral. Avaliação do perfil nutricional de crianças de um CMEI da região de Curitiba-PR. Cadernos da Escola de Saúde, v. 1, n. 9, 2017.

GÓES, JAW et al. Capacitação dos manipuladores de alimentos e a qualidade da alimentação servida.Higiene Alimentar, São Paulo, v. 15, n. 82, p. 20-22, mar, 2001.

GOULART, Lúcia Maria Horta de Figueiredo; VIANA, Maria Regina de Almeida. Saúde da criança e do adolescente: agravos nutricionais. 2008.

HENRIQUES, Bruno José Monteiro. Relação entre a higienização de manipuladores e 
superfícies e a contaminação do produto final em pequenas indústrias alimentares. 2014 . Dissertação de Mestrado. Universidade de Aveiro.

MULLER, Boas Práticas de Manipulação de Alimentos com Merendeiras,UNIVERSIDADE DO OESTE DE SANTA CATARINA - UNOESC Especialização de Microbiologia Industrial e de Alimentos, 2011.

OLIVEIRA, Mariana de Novaes; BRASIL, Anne Lise Dias; TADDEI, José Augusto de Aguiar Carrazedo. Avaliação das condições higiênico-sanitárias das cozinhas de creches públicas e filantrópicas. Ciência \& Saúde Coletiva, 2008.

RESOluÇÃO, R. D. C. nº. 21, de 26 de janeiro de 2001, Brasil. Agência Nacional de Vigilância Sanitária. Aprova o Regulamento Técnico para Irradiação de Alimentos. Diário Oficial da União, Brasília, n. 20-E, p. 35.

RIEKES, Bethania Hering et al. Qualidade em unidades de alimentação e nutrição: uma proposta metodológica considerando aspectos nutricionais e sensoriais. 2004.

ROCHA, Bárbara et al. Avaliação das condições higiênico-sanitárias e da temperatura das refeições servidas em restaurantes comerciais do tipo self-service. Perquirere, v. 1, n. 7, p. 30-40, 2010.

SILVA, Gilmar; OLIVEIRA, Ana Cristina; SANTOS, Ondina. Avaliação das condições higiênico-sanitárias da cozinha do CMEI do município de Rialma-GO. JIC-Jornada de Pesquisa e Iniciação Científica, v. 3, n. 3, 2012.

SILVA LC, Santos DB, São José JFB, Silva EMM. Boas práticas na manipulação de alimentos em Unidades de Alimentação e Nutrição. Demetra 2015; 10(4):797-820.

SOUZA, C. H. et al. Avaliação das condições higiênico sanitárias em uma unidade de alimentação e nutrição hoteleira, na cidade de Timóteo-MG. Nutrir Gerais, v. 3, n. 4, p. 31229, 2009.

STEFANELLO, Cláudia Luísa; LINN, Débora Schmidt; MESQUITA, M. O. Percepção sobre Boas Práticas por cozinheiras e auxiliares de cozinha de uma UAN do Noroeste do Rio Grande do Sul. Vivências, v. 5, n. 8, p. 93-98, 2009.

VILA, Carla Vanez Dias; SILVEIRA, Joice Trindade; ALMEIDA, Lana Carneiro. Condições higiênico-sanitárias de cozinhas de escolas públicas de Itaqui, Rio Grande do Sul, Brasil. Vigilância Sanitária em Debate: Sociedade, Ciência \& Tecnologia, v. 2, n. 2, p. 6774, 2014.

VOOS, Ana Carolina. Atuação do nutricionista no Programa Nacional de Alimentação Escolar (PNAE) no estado do RS. 2009. 